\title{
ORIGINAL RESEARCH \\ Association of Hemodynamic Characteristics and Cerebral Aneurysm Rupture
}

J.R. Cebral

F. Mut

J. Weir

C.M. Putman

BACKGROUND AND PURPOSE: Hemodynamic factors are thought to play an important role in the initiation, growth, and rupture of cerebral aneurysms. This report describes a study of the associations between qualitative intra-aneurysmal hemodynamics and the rupture of cerebral aneurysms.

MATERIALS AND METHODS: Two hundred ten consecutive aneurysms were analyzed by using patientspecific CFD simulations under pulsatile flow conditions. The aneurysms were classified into categories by 2 blinded observers, depending on the complexity and stability of the flow pattern, size of the impingement region, and inflow concentration. A statistical analysis was then performed with respect to the history of previous rupture. Interobserver variability analysis was performed.

RESULTS: Ruptured aneurysms were more likely to have complex flow patterns (83\%, $P<.001$ ), stable flow patterns $(75 \%, P=.0018)$, concentrated inflow $(66 \%, P=<.0001)$, and small impingement regions $(76 \%, P=.0006)$ compared with unruptured aneurysms. Interobserver variability analyses indicated that all the classifications performed were in very good agreement-that is, well within the $95 \% \mathrm{Cl}$.

CONCLUSIONS: A qualitative hemodynamic analysis of cerebral aneurysms by using image-based patient-specific geometries has shown that concentrated inflow jets, small impingement regions, complex flow patterns, and unstable flow patterns are correlated with a clinical history of prior aneurysm rupture. These qualitative measures provide a starting point for more sophisticated quantitative analysis aimed at assigning aneurysm risk of future rupture. These analyses highlight the potential for CFD to play an important role in the clinical determination of aneurysm risks.

ABBREVIATIONS: $\mathrm{ACA}=$ anterior cerebral artery; $\mathrm{AcomA}=$ anterior communicating artery; $\mathrm{C}=$ complex (flow complexity), concentrated (inflow concentration); CFD = computational fluid dynamics; $\mathrm{Cl}=$ confidence interval; $\mathrm{D}=$ diffuse; $3 \mathrm{DRA}=3 \mathrm{D}$ rotational angiography; $\mathrm{L}=$ large; $\mathrm{OR}=$ odds ratio; $\mathrm{S}=$ simple (flow complexity), stable (flow stability), or small (flow impingement); 01-O2 = observer 1-observer 2; $U$ = unstable; $V=$ velocity; WSS = wall sheer stress

A lthough unruptured cerebral aneurysms carry a relatively low risk of rupture, ${ }^{1,2}$ preventive interventions are commonly considered because of the poor prognosis of intracranial hemorrhage. Current treatments of intracranial aneurysms carry a small but significant risk that can exceed the natural risk of aneurysm rupture, ${ }^{2,3}$ making the development of methods to better define the rupture risk of cerebral aneurysms very valuable for clinicians. Current risk assessment is mainly based on aneurysm size - that is, larger aneurysms are more likely to rupture than smaller aneurysms. ${ }^{4,5}$ However, small aneurysms do rupture; thus, aneurysm size alone may not be enough for a reliable stratification of aneurysm rupture risk. Hemodynamics is commonly thought to play an important role in the mechanisms of aneurysm development, progression, and rupture. ${ }^{6-9}$ It is reasonable to assume that rup-

Received May 3, 2010; accepted after revision June 25.

From the Department of Computational and Data Sciences (J.R.C., F.M.), Center for Computational Fluid Dynamics, George Mason University, Fairfax, Virginia; and Department of Interventional Neuroradiology (J.W., C.M.P.), Inova Fairfax Hospital, Falls Church, Virginia.

This work was supported by Philips Healthcare and a National Institutes of Health grant (R01NS059063).

Please address correspondence to Juan R. Cebral, PhD, Center for Computational Fluid Dynamics, Department of Computational and Data Sciences, George Mason University, 4400 University Dr, MSN 6A2, Fairfax, VA 22030; e-mail: jcebral@gmu.edu

Indicates open access to non-subscribers at www.ajnr.org

DOI 10.3174/ajnr.A2274 ture risk assessment can be improved by incorporating hemodynamic information. Several researchers have considered number of geometric measures, such as aspect ratio and shape descriptors, as surrogates for hemodynamic information. ${ }^{10-13}$ These parameters may be useful for improving risk evaluation, but they are difficult to connect to the underlying mechanisms. Others have focused on extracting hemodynamic information from CFD models. ${ }^{14-16}$ So far, these studies have been limited to a small number of aneurysms, which has prevented the establishment of statistical associations between hemodynamic characteristics and aneurysm rupture. The purpose of this study was to statistically confirm previous trends relating qualitative hemodynamic characteristics and aneurysm rupture by using image-based CFD analysis. ${ }^{17}$

\section{Materials and Methods}

\section{Patients and Aneurysms}

A total of 210 intracranial aneurysms in 128 consecutive patients referred to the Interventional Neuroradiology Service and imaged by conventional catheter angiography and 3DRA were included in this study. Patient ranged from 28 to 88 years of age, with a mean age of 54 years. Women accounted for $68 \%$ of the patients. The patients' medical and radiologic records were reviewed and evaluated for evidence of aneurysmal intracranial hemorrhage. In patients with multiple aneurysms, the clinical and radiologic information was considered, and a judgment of the most likely source of hemorrhage was made. The other coincident aneurysms were classified as unruptured. Dissecting 
aneurysms or aneurysms with inconclusive clinical information or with evidence of vasospasm were excluded. In addition to the symptoms associated with the intracranial hemorrhage in patients with ruptured aneurysms, a relatively small number of patients (10\%) presented with a variety of symptoms, including the following: aphasia (3 patients), bilateral peripheral weakness (1 patient), cranial nerve III palsy (4 patients), right neck pain (1 patient), right-sided weakness ( 2 patients), brain arteriovenous malformations (2 patients), and seizure disorder (1 patient). The aneurysms were distributed in various locations: 53 in the internal carotid artery, 39 in the middle cerebral artery, 52 in the posterior communicating artery, 28 in the AcomA, 4 in the ACA, 26 in the basilar artery, 2 in the superior cerebellar artery, 1 in the posterior cerebral artery, and 5 in the vertebral artery. A total of $176(83 \%)$ aneurysms were located in the anterior circulation, with 34 (17\%), in the posterior circulation. The distribution of aneurysms among morphologic categories was as follows: 67 bifurcation aneurysms, 69 terminal aneurysms, 59 lateral aneurysms, 12 bilateral AcomA aneurysms, and 3 fusiform aneurysms.

\section{Image Data}

All catheter angiograms were performed by standard transfemoral catheterizations of the cerebral blood vessels. Digital subtraction angiography imaging was performed on an Integris biplane unit (Philips Healthcare, Best, the Netherlands). Rotational angiograms were performed during a 6-second contrast injection for a total of $24 \mathrm{~mL}$ of contrast agent and a $180^{\circ}$ rotation imaging at 15 frames per second during 8 seconds, for an acquisition of 120 projection images. Bilateral 3DRA images were acquired for aneurysms in the AcomA artery accepting blood from both A1 segments of the ACAs. The projection images were transferred to the Integris 3DRA Workstation (Philips Healthcare) and reconstructed into 3D voxel data by using standard proprietary software (XtraVision, Philips Healthcare).

\section{Vascular and Hemodynamics Modeling}

Patient-specific models of the cerebral aneurysms were constructed by using a previously developed methodology. ${ }^{18,19}$ Briefly, 3D images were filtered to reduce noise and segmented by using a seeded regiongrowing algorithm to reconstruct the arterial network topology followed by an isosurface deformable model to recover the vascular geometry. The vascular models were then smoothed, and vessel branches were truncated perpendicularly to their axes. Unstructured grids composed of tetrahedral elements were then generated for numeric simulations with a minimum uniform resolution between 0.02 $\mathrm{cm}$ and $0.01 \mathrm{~cm}$. The resulting grids contained between 1 and 5 million elements.

Blood flows were approximated by the unsteady 3D Navier-Stokes equations for an incompressible Newtonian fluid, and vessel walls were assumed rigid. Because patient-specific blood-flow information was not available, typical physiologic flow boundary conditions were derived from phase-contrast MR imaging measurements of flow rates in healthy subjects. ${ }^{20}$ The measured flow waveforms were scaled with the areas of the inlet boundaries to achieve a mean WSS of 15 dyne/ $\mathrm{cm}^{2}$ at the inlets, which were located in the internal carotid artery, vertebral artery, or basilar artery for all models. Fully developed velocity profiles were prescribed at the inlets by using the Womersley solution. ${ }^{21}$ The governing equations were numerically solved by using in-house-developed software based on an implicit pressure-projection algorithm. ${ }^{22,23}$ All numeric simulations were performed by using 100 time steps per cardiac cycle for a total of 2 cycles. All analyses and visualizations were done for the second cycle.

\section{Data Analysis}

The computed blood flow fields were visualized by using a variety of techniques, including the following: 1) isovelocity surfaces to depict the aneurysm inflow stream, 2) streamlines to depict the intra-aneurysmal flow structures, 3 ) velocity magnitudes on cut planes to depict the inflow jets and velocity profiles at the neck, and 4) shaded surfaces to depict the distribution of WSS magnitudes.

The hemodynamic visualizations were analyzed to classify blood flows according the following characteristics:

Flow complexity. "Simple" flow pattern indicates flow patterns consisting of a single recirculation zone or vortex structure within the aneurysm. "Complex" indicates flow patterns exhibiting flow divisions or separations within the aneurysm sac and containing more than 1 recirculation zone or vortex structure.

Flow stability. "Stable" indicates flows patterns that persist (do not move or change) during the cardiac cycle. "Unstable" indicates flow patterns in which the flow divisions and/or vortex structures move or are created or destroyed during the cardiac cycle.

Inflow concentration. "Concentrated" inflow streams or jets penetrate relatively deep into the aneurysm sac and are thin or narrow in the main flow direction. "Diffuse" indicates inflow streams that are thick compared with the aneurysm neck and flow jets that disperse quickly once they penetrate into the aneurysm sac.

Flow impingement. The "flow impingement zone" is the region of the aneurysm where the inflow stream is seen to impact the aneurysm wall and change its direction and/or disperse. Typically this region has an associated region of elevated WSS: a small impingement if the area of the impingement region is small compared with the area of the aneurysm $(<50 \%)$; a large impingement, if the area of impingement is large compared with the area of the aneurysm $(>50 \%)$.

Two observers blinded to the clinical history of the patients independently evaluated and classified the aneurysms into the categories described above. The degree of agreement between the 2 observers was quantified by using the $\kappa$ test. ${ }^{24}$ In cases in which the observers disagreed, the models were re-examined by both observers and a consensus was reached. The data were then analyzed to look for associations between the hemodynamic characteristics and the clinical history of aneurysm rupture. Statistical analysis was performed by using $2 \times 2$ contingency tables, and 2 -tailed $P$ values were calculated by using the $\chi^{2}$ (Pearson uncorrected) test. ${ }^{24}$ Associations were considered statistically significant if the $P$ values were $<.05$ (95\% CI).

\section{Results}

Numeric simulations were performed on the 210 patient-specific aneurysm geometries under pulsatile flows. Visualizations of the unsteady flow fields were composed of cine-loops and were used to classify the aneurysms into the hemodynamic categories described earlier. Examples of aneurysms with simple and complex flow patterns are shown in Fig 1. Examples of aneurysms with stable and unstable flow patterns are shown in Fig 2. Examples of aneurysms with large and small impingement regions are shown in Fig 3. Examples of aneurysms with diffuse and concentrated inflow streams are shown in Fig 4.

The number of aneurysms classified into each category by each observer was counted and used to assess the degree of agreement of the hemodynamic classification. The results are presented in Table 1. This table shows, for each hemodynamic characteristic, the agreement-disagreement table, the number of aneurysms that were classified by both observers into the 

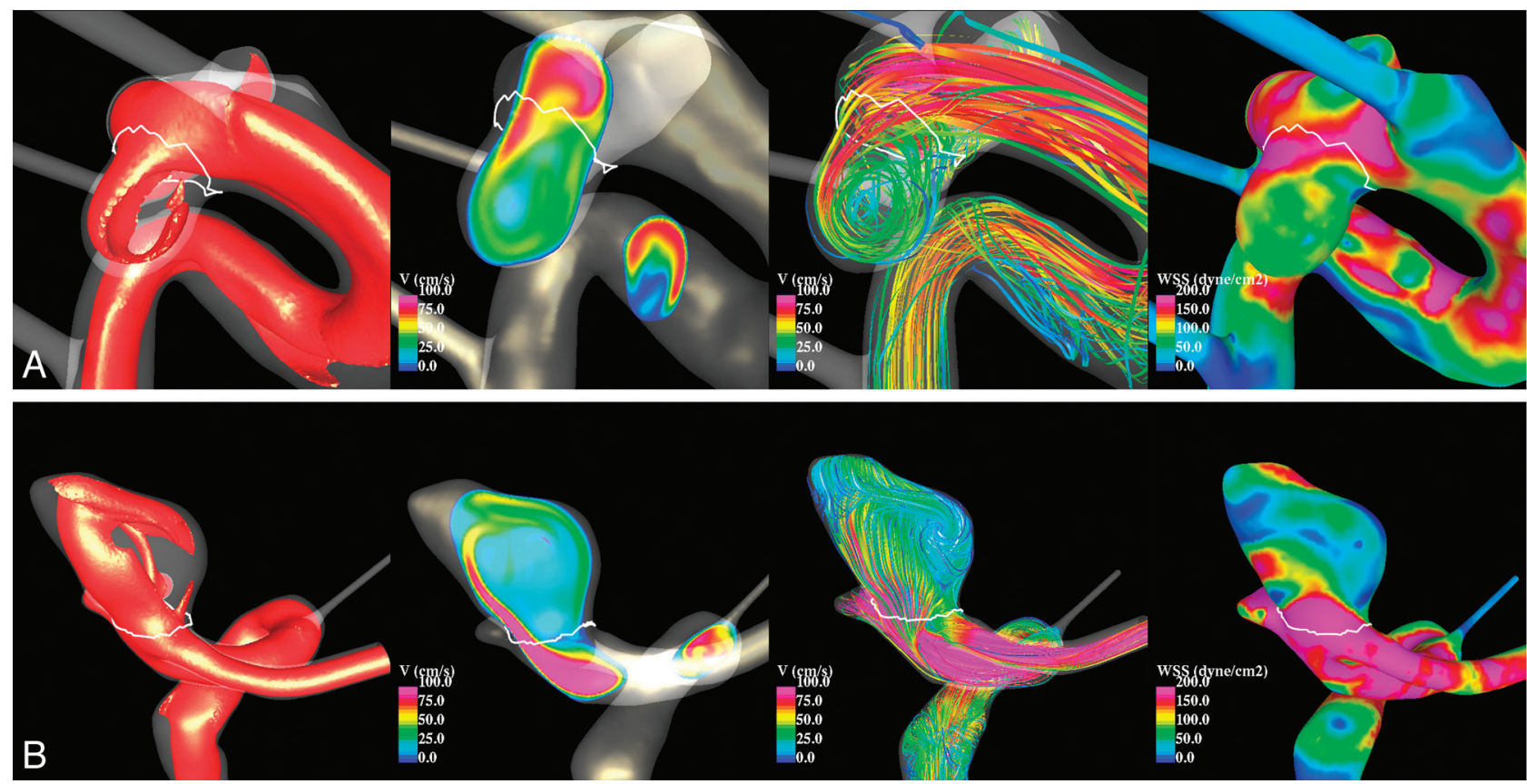

Fig 1. Examples of aneurysms with simple $(A)$ and complex $(B)$ flow patterns. From left to right, the visualizations show isovelocity surfaces, velocity magnitudes on a cut plane, streamlines, and WSS distribution, all at peak systole.

same categories (agreements), the number of agreements expected by chance (random), the $\kappa$ value, and the $95 \%$ CI. The agreement-disagreement tables list the number of aneurysms that were classified into the same category by both observers and the number of aneurysms classified into 1 category by observer 1 and into another by observer 2 . The $\kappa$ values obtained indicate that all the classifications performed by both observers were in very good agreement-that is, well within the $95 \%$ CI.

The number of ruptured and unruptured aneurysms in each category was then counted. These numbers along with the results of the statistical analyses by using contingency tables are presented in Table 2. This table also lists the $\chi^{2}$ values, 2 -tailed $P$ values, and ORs computed from the $2 \times 2$ contingency tables. The ORs indicate that ruptured aneurysms are 4.701 times more likely to have complex flow patterns, 2.733 times more likely to have unstable flow patterns, 3.975 more likely to have concentrated inflows, and 3.005 more likely to have small impingement regions. The corresponding $P$ values show that these associations reached a strong statistical significance, well above the 95\% CI. On the other hand, unruptured aneurysms are more likely to have diffuse inflows, but they are roughly equally likely to have simple or complex, and stable or unstable flow patterns, and large or small impingement regions. In addition, the average size (maximum dome diameter) of the ruptured-aneurysm group $(9.3 \mathrm{~mm})$ was larger than that of the unruptured aneurysm group $(6.5 \mathrm{~mm})$. This difference was statistically significant, according to the Student $t$ test with a $95 \%$ CI.

\section{Discussion}

The pathophysiology of cerebral aneurysms is complex and poorly understood. Current theories implicate genetic factors, perianeurysmal environment, and vascular wall biology in combination with the hemodynamic environment as the de- terminants of whether aneurysms will progress and ultimately rupture. ${ }^{7}$ Although intravascular hemodynamics is widely considered important in the process, there is no consensus on which variables are most important. Great controversy exists as to whether regions of low or high flow are the most critical in promoting the events responsible for rupture. The presence of a low-flow environment could potentially lead to changes in the arterial wall that would weaken its structural integrity through mechanisms related to wall inflammation. Low flows lead to regions of low WSS, which can be detrimental to the wall endothelium. In particular, stagnant blood flow promotes thrombus formation which, when adjacent to the aneurysm wall, can lead to the release of substances that promote inflammation in the aneurysm wall. ${ }^{25-27}$ Inflammation can be associated with structural degradation through the release of numerous types of destructive enzymes. ${ }^{28,29}$

High intravascular blood flow causes an elevation of WSS. At high levels of WSS, the endothelium releases nitrous oxide that leads to remodeling of the arterial wall in a system that seeks to maintain the WSS within an acceptable range. ${ }^{30-32}$ At excessive levels of WSS, the endothelium becomes dysfunctional and can be destroyed. ${ }^{33}$ Determining which hemodynamic variables are most closely correlated to clinical progression and rupture may help us determine the relative influence of these mechanisms. This knowledge may help in developing further improvements in our treatment paradigms for cerebral aneurysms.

Cebral et $\mathrm{al}^{17}$ reported a series of 62 aneurysms in which CFD analysis was performed on patient-specific models and CFD findings were correlated with a clinical history of prior rupture. They found that unruptured aneurysms more commonly had simple stable flow patterns, large impingement regions, and large jet sizes, while ruptured aneurysms had disturbed flow patterns, small impingement regions, and narrow jets. Of these characteristics, only impingement size reached 

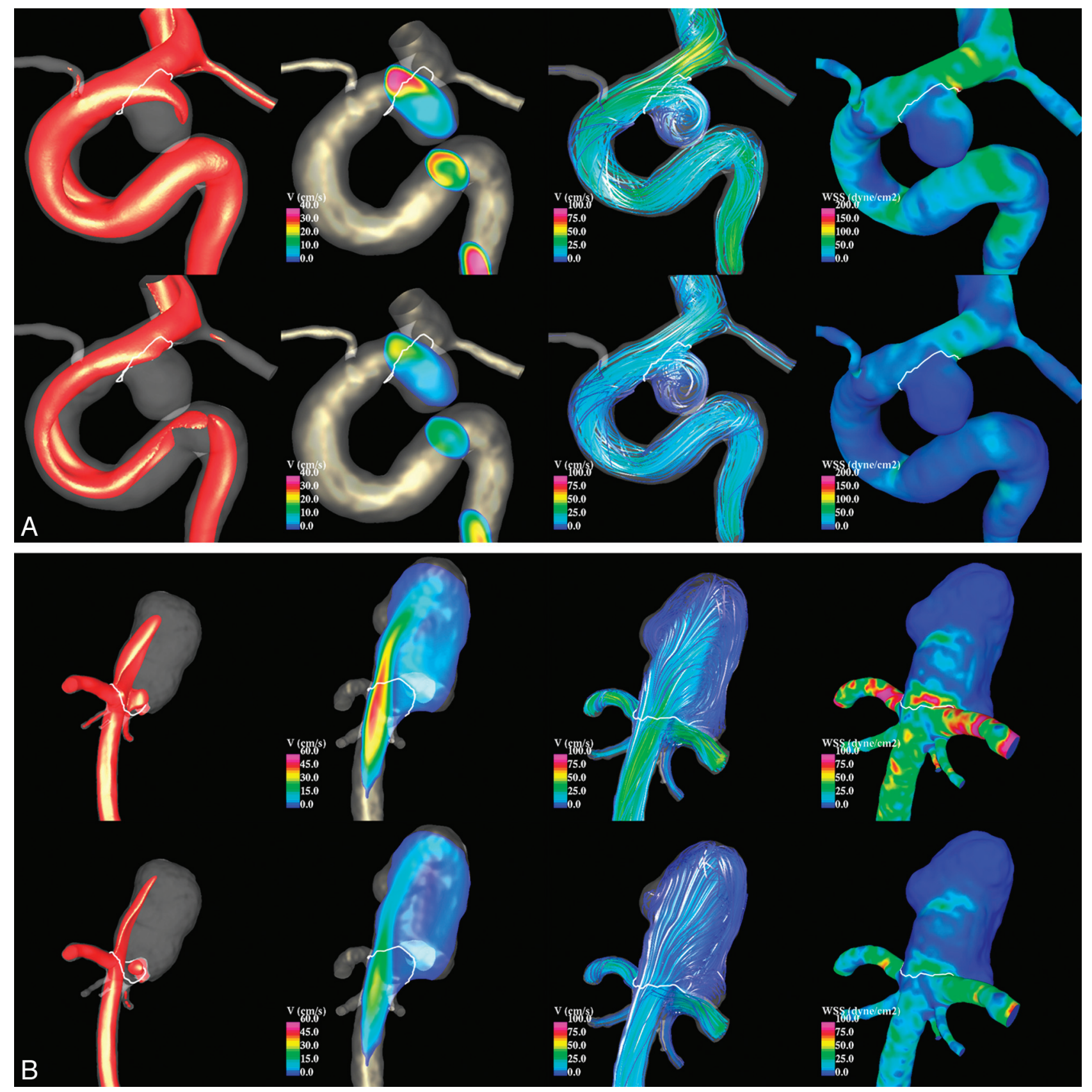

Fig 2. Examples of aneurysms with stable $(A)$ and unstable $(B)$ flow patterns. Visualizations at peak systole (top row of each panel) and end diastole (bottom row of each panel) are shown by using (from left to right) isovelocity surfaces, velocity magnitudes on a cut plane, streamlines, and WSS distribution.

statistical significance, possibly due to the small sample size. The current study focuses on confirming the previous trends observed. Our study incorporates a number of improvements and extensions to previously reported analyses. First, the sample was large enough to achieve statistically significant results. Second, the aneurysm population included aneurysms in the anterior communicating complex, which had been previously excluded because not all avenues of flow had been properly imaged and therefore did not allow proper CFD modeling. Third, the classification of intra-aneurysmal flow patterns was simplified by considering flow complexity and flow stability separately. These 2 characteristics now have become dichotomous variables, which make both the aneurysm classification and subsequent statistical analysis simpler and more robust.
Finally, the current study includes an interobserver variability analysis showing a high degree of agreement between observers classifying aneurysms into the proposed hemodynamic categories.

The statistical analysis indicates that the qualitative hemodynamic characteristics considered are strongly correlated with aneurysm rupture-namely, that ruptured aneurysms are more likely to have complex and unstable flows, concentrated inflows, and small impingement regions. While most unruptured aneurysms had diffuse inflows, many of them had complex flows, unstable flows, and/or small impingement regions. From the mechanistic perspective, the results seem to point to regions of concentrated, more rapid flow as correlating with rupture rather than implicating the presence of a 

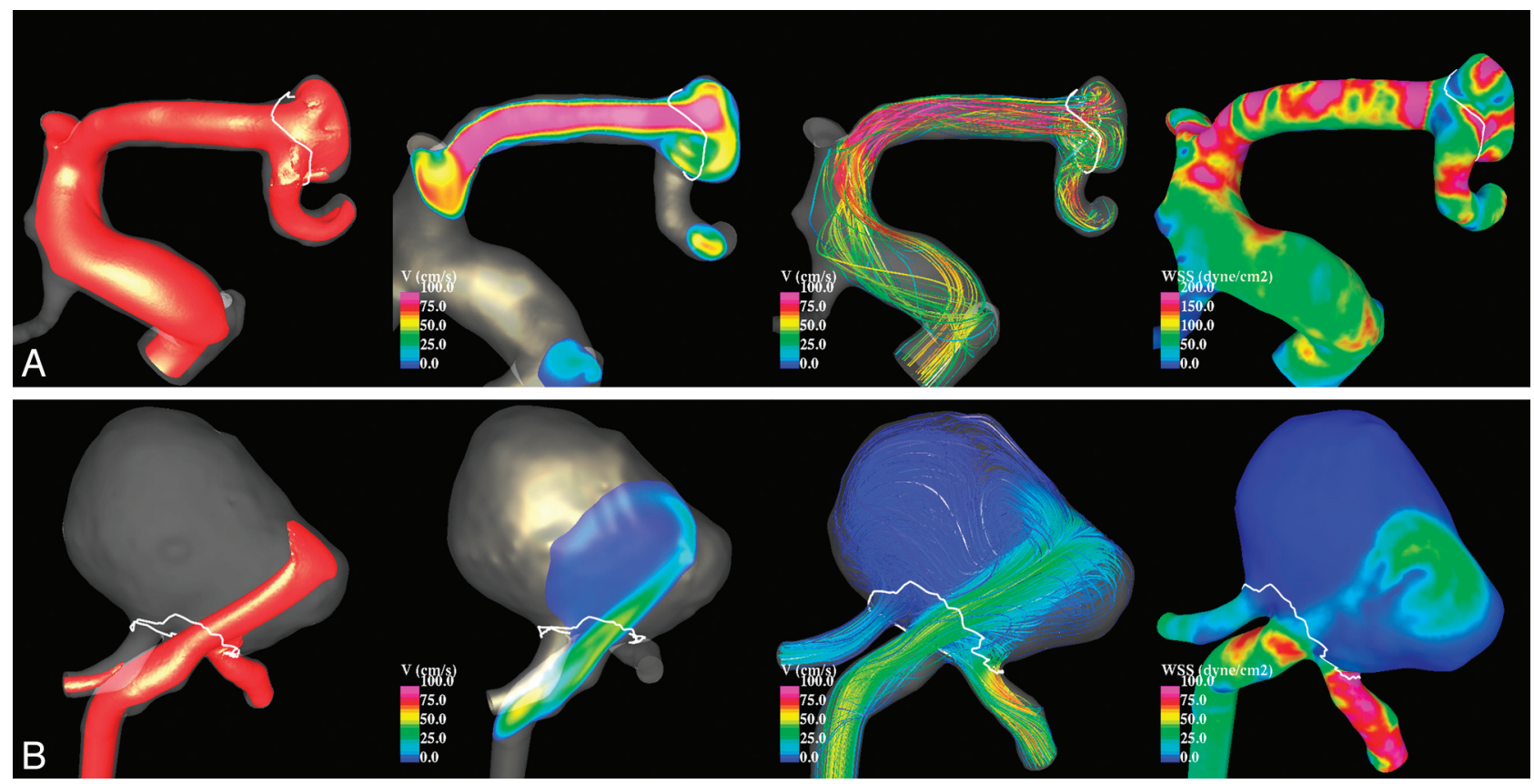

Fig 3. Examples of aneurysms with large $(A)$ and small $(B)$ impingement regions. From left to right, the visualizations show isovelocity surfaces, velocity magnitudes on a cut plane streamlines, and WSS distribution, all at peak systole.
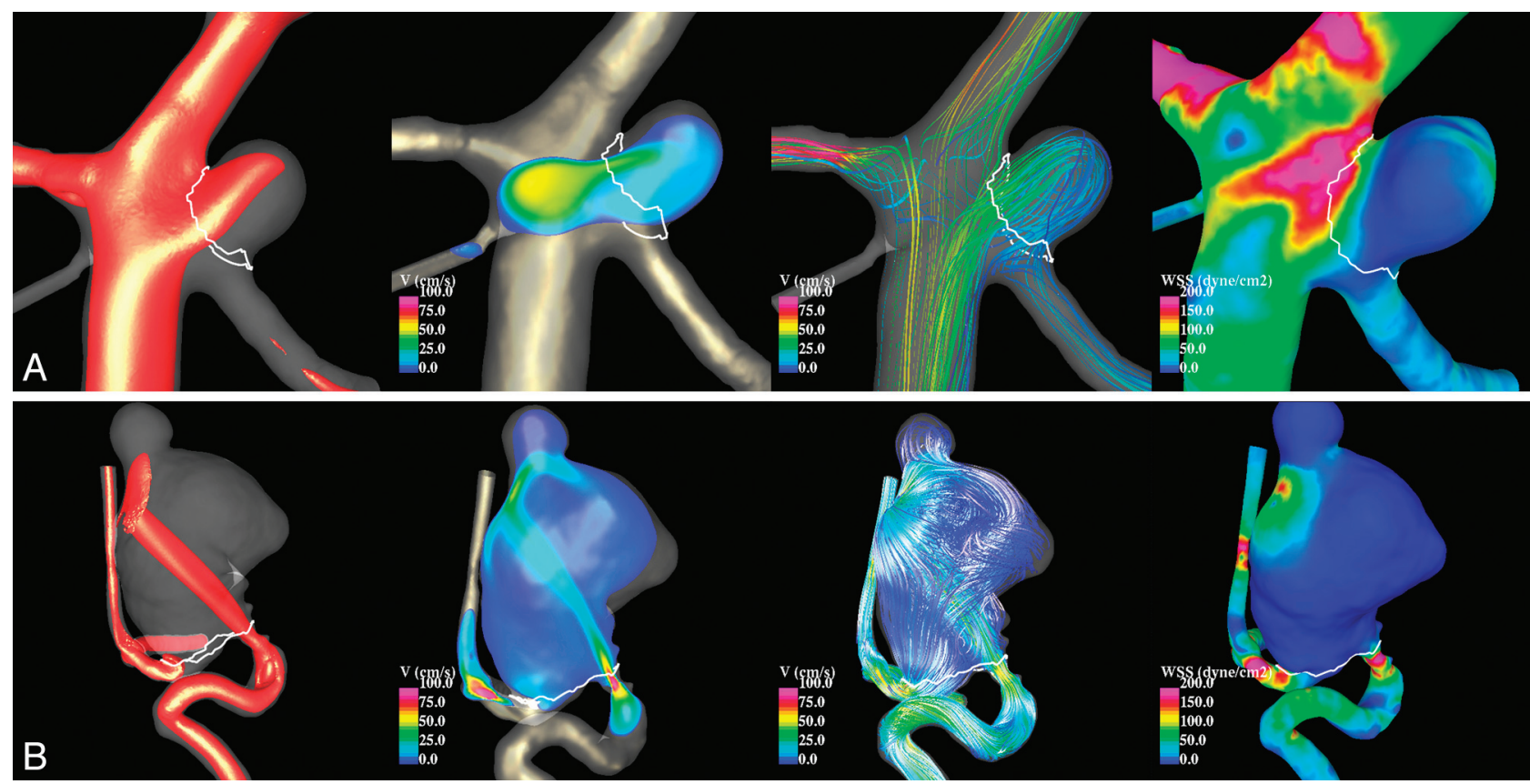

Fig 4. Examples of aneurysms with diffuse $(A)$ and concentrated $(B)$ inflows. From left to right, the visualizations show isovelocity surfaces, velocity magnitudes on a cut plane, streamlines, and WSS distribution, all at peak systole.

low-flow environment. However, the simplified qualitative analysis performed in this study does not specifically seek to examine the probably complex interrelationship between lowand high-flow hemodynamic variables. This would require a more sophisticated quantitative multivariate analysis before any strong conclusions should be drawn.

It is not surprising that both categories of aneurysms are found in the unruptured group because this analysis is an examination of only a single point in time without a complete knowledge of the final outcome of each aneurysm. Many unruptured aneurysms may progress with time and become rup- tured. Therefore, many of the unruptured aneurysms with complex and unstable flows, concentrated inflows, and small impingement regions may join the ruptured category, leaving a predominance of aneurysms with simpler, less concentrated flows. A longitudinal study of these aneurysms would be necessary to confirm this possibility. If confirmed, these CFD characterizations could potentially improve our ability to assign risk to an individual aneurysm and thereby improve the judgments made on the need for treatment of asymptomatic unruptured aneurysms.

The current study has a number of limitations that should 


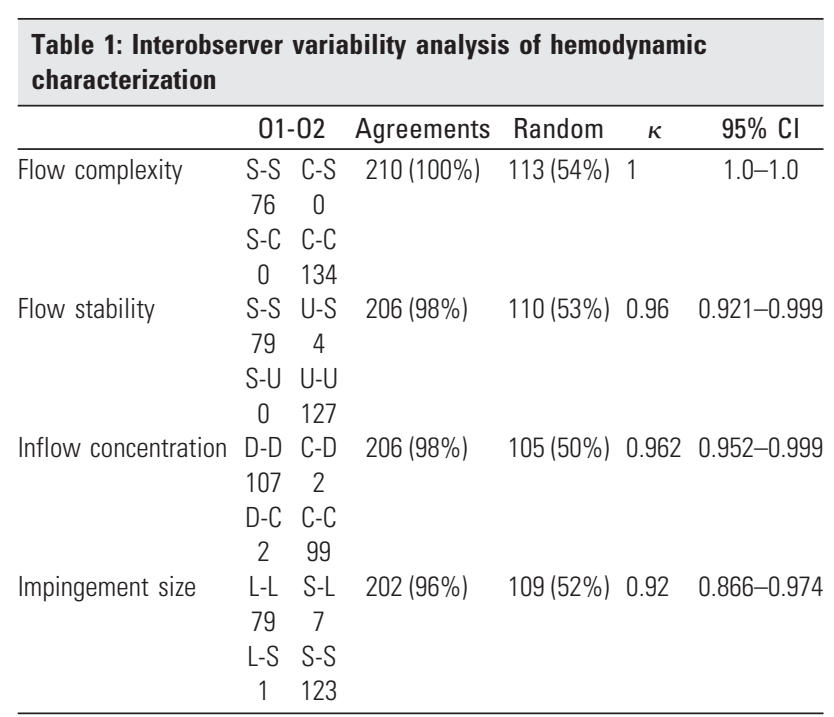

\begin{tabular}{|c|c|c|c|c|c|}
\hline & Unruptured & Ruptured & $\chi^{2}$ & $P$ & OR \\
\hline \multicolumn{6}{|l|}{ Flow complexity } \\
\hline Simple & 62 & 14 & 22.190 & $<.0001$ & 4.701 \\
\hline Complex & 65 & 69 & & & \\
\hline \multicolumn{6}{|l|}{ Flow stability } \\
\hline Stable & 59 & 20 & 10.694 & .0018 & 2.733 \\
\hline Unstable & 68 & 63 & & & \\
\hline \multicolumn{6}{|c|}{ Inflow concentration } \\
\hline Diffuse & 85 & 28 & 22.252 & $<.0001$ & 3.975 \\
\hline Concentrated & 42 & 55 & & & \\
\hline \multicolumn{6}{|l|}{ Impingement size } \\
\hline Large & 62 & 20 & 12.890 & .0006 & 3.005 \\
\hline Small & 65 & 63 & & & \\
\hline
\end{tabular}

be considered when interpreting the results. Similar to previous studies focusing on geometric characteristics of cerebral aneurysms (which are the basis of current rupture-risk assessment), in the current study, a retrospective analysis of a population including both unruptured and ruptured aneurysms was performed. This does not allow us to confirm whether unruptured aneurysms in the high-risk categories (those more likely to occur in ruptured aneurysms) will indeed rupture. However, recent case studies of cerebral aneurysms imaged just before their rupture are in very good agreement with the predictions of our current study. ${ }^{34,35}$ In addition, this study relies on an assumption that the aneurysm anatomy is little changed by the event of rupture; however, an aneurysm may undergo a variety of structural changes during and immediately after a hemorrhage. For example, a portion of the aneurysm may be filled with thrombus or a new daughter sac may form. Small changes in the geometry can have significant effects on intra-aneurysmal patterns. Therefore, it may be necessary to conduct prospective natural history studies of CFDanalyzed unruptured aneurysms to evaluate conclusively the association of a predetermined hemodynamic factor to risk of aneurysmal rupture.

As in most CFD analyses, a number of assumptions and approximations were made during the modeling process. These include the following: Blood was modeled as a Newtonian fluid, vessel wall compliance was neglected, physiologic flow conditions were not patient-specific but derived from flow measurements in the cerebral arteries of healthy subjects, and the vascular geometries were approximated from 3D images with limited resolution. Previous sensitivity analyses by using a small number of aneurysm models suggested that the most important factor for a realistic representation of the in vivo hemodynamics is the vascular geometry. ${ }^{18,36}$ With different flow conditions, ${ }^{37}$ non-Newtonian viscosity models or compliant models ${ }^{38}$ did not substantially affect the qualitative hemodynamic characteristics. In the current study, careful attention was paid to the reconstruction of vascular models from the 3DRA images. Images that failed to properly depict the parent vessel because of incomplete filling or images that were too noisy due to low contrast dose were discarded. The entire portion of the proximal parent artery visible in the images was included in the models to properly capture the secondary and swirling flows created by the curving geometry of the parent vessel. Despite all these limitations and approximations, it has been shown that these CFD models are capable of realistically representing the in vivo intra-aneurysmal hemodynamic patterns observed with conventional angiography. ${ }^{39}$

During the past decade, great progress has been made in image-based CFD modeling of blood flows. Simulation software has become more accessible and easier to use. However, these techniques are still challenging, and models must be constructed carefully. The choice of boundary conditions, mesh and time resolution, segmentation methods and parameters, location of vessel truncation, inclusion of side arterial branches, and so forth can affect the quantitative hemodynamic results. For this reason, before attempting the quantification of hemodynamic variables, we proposed a qualitative characterization of aneurysmal flows based on observations of gross flow features and we investigated possible relationships with aneurysm rupture. The current study showed that these qualitative characteristics are indeed related to aneurysm rupture and thus justify the search for quantitative variables that objectively describe these hemodynamic categories. Perhaps some of the gross hemodynamic characteristics could be determined by using simplified models in an efficient manner, for instance by using steady flows, truncated models, coarse computational grids, and so forth. This could allow a quick computerized clinical evaluation of cerebral aneurysms and could potentially improve current patient management.

\section{Conclusions}

A qualitative hemodynamic analysis of cerebral aneurysms by using image-based patient-specific geometries has shown that concentrated inflow jets, small impingement regions, complex flow patterns, and unstable flow patterns are correlated with a clinical history of prior aneurysm rupture. These qualitative measures provide a starting point for more sophisticated quantitative analysis aimed at assigning aneurysm risk of future rupture. These analyses highlight the potential for CFD to play an important role in the clinical determination of aneurysm risks.

\section{References}

1. Kassell NF, Torner JC, Haley EC, et al. The International Cooperative Study On The Timing Of Aneurysm Surgery. Part 1. Overall management results. J Neurosurg 1990;73:37-47

2. Wiebers DO, Whisnant JP, Huston Jr, et al, for the International Study of Un- 
ruptured Intracranial Aneurysms Investigators. Unruptured intracranial aneurysms: natural history, clinical outcome, and risks of surgical and endovascular treatment. Lancet 2003;362:103-10

3. Tomasello F, D'Avella D, Salpietro FM, et al. Asymptomatic aneurysms: literature meta-analysis and indications for treatment. J Neurosurg Sci 1998;42:47-51

4. Nishioka H, Torner JC, Graf CJ, et al. Cooperative study of intracranial aneurysms and subarachnoid hemorrhage: a long-term prognostic study. II. Ruptured intracranial aneurysms managed conservatively. Arch Neurol 1984;41:1142-46

5. White PM, Wardlaw JM. Unruptured intracranial aneurysms. J Neuroradiol 2003;30:336-50

6. Stehbens WE. Intracranial aneurysms. In: Stebbens WE. Pathology of the Cerebral Blood Vessels. St. Louis: CV Mosby; 1972:351-470

7. Sforza D, Putman CM, Cebral JR. Hemodynamics of cerebral aneurysms. Annu Rev Fluid Mech 2009;41:91-107

8. Kayembe KN, Sasahara M, Hazama F. Cerebral aneurysms and variations of the circle of Willis. Stroke 1984;15:846-50

9. Nixon AM, Gunel M, Sumpio BE. The critical role of hemodynamics in the development of cerebral vascular disease. J Neurosurg 2010;112:1240-53

10. Ujiie $\mathrm{H}$, Tamano $\mathrm{Y}$, Sasaki $\mathrm{K}$, et al. Is the aspect ratio a reliable index for predicting the rupture of a saccular aneurysm? Neurosurgery 2001;48:495-503

11. Raghavan ML, Ma B, Harabaugh RE. Quantified aneurysm shape and rupture risk. J Neurosurg 2005;102:355-62

12. Ma B, Harbaugh RE, Raghavan ML. Three-dimensional geometrical characterization of cerebral aneurysms. Ann Biomed Eng 2004;32:264-73

13. Millán RD, Dempere-Marco L, Pozo JM, et al. Morphological characterization of intracranial aneurysms using 3-D moment invariants. EEE Trans Med Imaging 2007;26:1270-82

14. Shojima M, Oshima M, Takagi $K$, et al. Magnitude and role of wall shear stress on cerebral aneurysm: computational fluid dynamic study of 20 middle cerebral artery aneurysms. Stroke 2004;35:2500-05

15. Steinman DA, Milner JS, Norley CJ, et al. Image-based computational simulation of flow dynamics in a giant intracranial aneurysm. AJNR Am J Neuroradiol 2003;24:559-66

16. Jou LD, Quick CM, Young WL, et al. Computational approach to quantifying hemodynamic forces in giant cerebral aneurysms. AJNR Am J Neuroradiol 2003;24:1804-10

17. Cebral JR, Castro MA, Burgess JE, et al. Characterization of cerebral aneurysm for assessing risk of rupture using patient-specific computational hemodynamics models. AJNR Am J Neuroradiol 2005;26:2550-59

18. Cebral JR, Castro MA, Appanaboyina S, et al. Efficient pipeline for image-based patient-specific analysis of cerebral aneurysm hemodynamics: technique and sensitivity. EEE Trans Med Imaging 2005;24:457-67

19. Castro MA, Putman CM, Cebral JR. Patient-specific computational modeling of cerebral aneurysms with multiple avenues of flow from 3D rotational angiography images. Acad Radiol 2006;13:811-21

20. Cebral JR, Castro MA, Putman CM, et al. Flow-area relationship in internal carotid and vertebral arteries. Physiol Meas 2008;29:585-94
21. Taylor CA, Hughes TJR, Zarins CK. Finite element modeling of blood flow in arteries. Comput Methods Appl Mech Eng 1998;158:155-96

22. Löhner R. Applied CFD techniques. Hoboken, New Jersey: Wiley \& Sons; 2001

23. Mut F, Aubry R, Löhner R, et al. Fast numerical solutions in patient-specific simulations of arterial models. Int J Numer Meth Biomed Engng 2010; 26:73-85

24. Web Pages that Perform Statistical Calculations! http://www.Statpages.Org. Accessed February 2010

25. Griffith TM. Modulation of blood flow and tissue perfusion by endotheliumderived relaxing factor. Exp Physiol 1994;779:873-913

26. Moncada S, Plamer RM, Higgs EA. Nitric oxide: physiology, pathology and pharmacology. Pharmacol Rev 1991;43:109-42

27. Moritake K, Handa H, Hayashi K, et al. Experimental studies on intracrania aneurysms (a preliminary report): some biomechanical considerations on the wall structures of intracranial aneurysms and experimentally produced aneurysms. No Shinkei Geka 1973;1:115-23

28. Crawford T. Some observations of the pathogenesis and natural history of intracranial aneurysms. J Neurol Neurosurg Psychiatry 1959;22:259-66

29. Crompton M. Mechanism of growth and rupture in cerebral berry aneurysms. BMJ 1966;1:1138-42

30. Sho E, Sho M, Singh TM, et al. Blood flow decrease induces apoptosis of endothelial cells in previously dilated arteries resulting from chromic high blood flow. Arterioscler Thromb Vasc Biol 2001;21:1139-45

31. Hara A, Yoshimi N, Mori H. Evidence for apoptosis in human intracranial aneurysms. Neurol Res 1998;20:127-30

32. Fukuda S, Hashimoto N, Naritomi H, et al. Prevention of rat cerebral aneurysm formation by inhibition of nitric oxide synthase. Circulation 2000;101:2532-38

33. Nakatani H, Hashimoto N, Kang Y, et al. Cerebral blood flow patterns at major vessel bifurcations and aneurysms in rats. J Neurosurg 1991;74:258-62

34. Cebral JR, Hendrickson S, Putman CM. Hemodynamics in a lethal basilar artery aneurysm just before its rupture. AJNR Am J Neuroradiol 2009;30:95-98

35. Sforza D, Putman CM, Scrivano E, et al. Blood flow characteristics in a terminal basilar tip aneurysm prior to its fatal rupture. AJNR Am J Neuroradiol 2010;31:1127-31. Epub 2010 Feb 11

36. Castro MA, Putman CM, Cebral JR. Computational fluid dynamics modeling of intracranial aneurysms: effects of parent artery segmentation on intra-aneurysmal hemodynamics. AJNR Am J Neuroradiol 2006;27:1703-09

37. Castro MA, Putman CM, Cebral JR. Patient-specific computational fluid dynamics modeling of anterior communicating artery aneurysms: a study of the sensitivity of intra-aneurysmal flow patterns to flow conditions in the carotid arteries. AJNR Am J Neuroradiol 2006;27:2061-68

38. Dempere-Marco L, Oubel E, Castro MA, et al. CFD analysis incorporating the influence of wall motion: application to intracranial aneurysms. Med Image Comput Comput Assist Interv 2006;9:438-45

39. Cebral JR, Pergolizzi R, Putman CM. Computational fluid dynamics modeling of intracranial aneurysms: qualitative comparison with cerebral angiography. Acad Radiol 2007;14:804-13 